\title{
Automatic laser beam characterization of monolithic Nd:YAG nonplanar ring lasers
}

\author{
Patrick Kwee and Benno Willke \\ Max-Planck-Institut für Gravitationsphysik (Albert-Einstein-Institut) and Leibniz Universität Hannover, \\ 30167 Hannover, Germany \\ *Corresponding author: patrick.kwee@aei.mpg.de
}

Received 25 August 2008; revised 3 October 2008; accepted 6 October 2008; posted 9 October 2008 (Doc. ID 100593); published 5 November 2008

\begin{abstract}
A detailed beam characterization of continuous-wave single-frequency Nd:YAG solid-state ring lasers at a wavelength of $1064 \mathrm{~nm}$ is presented. The power noise, frequency noise, beam pointing fluctuations, spatial beam quality, and other properties of eight lasers of the same model were measured with a compact diagnostic instrument based on an optical ring resonator. One of the eight lasers was automatically characterized over a period of 3.5 months to investigate the long-term behavior. The results show that these lasers are highly stable laser sources, that the variations between different samples are rather small, and that these lasers are ideally suited for high precision optical experiments. (C) 2008 Optical Society of America
\end{abstract}

OCIS codes: $\quad 140.3295,140.3530,230.5750$.

\section{Introduction}

Many optical experiments require laser sources with exceptional beam properties. Especially precision experiments such as interferometric gravitational wave detectors [1-4] or experiments in quantum optics $[5,6]$ have strict requirements for the output beam of their laser systems. Therefore it is often necessary to stabilize several laser beam properties, such as the power [7], frequency [8], and beam pointing, by feedback control systems or filter resonators in order to fulfill the requirements. To design such stabilizations, the laser beam and appropriate actuators have to be characterized first.

A continuous-wave single-frequency monolithic nonplanar ring oscillator (NPRO) $[9,10]$ at a wavelength of $1064 \mathrm{~nm}$ is frequently used in high precision experiments. This kind of laser has a high intrinsic frequency stability since its resonator is built from a monolithic neodymium-doped yttrium aluminum garnet (Nd:YAG) crystal.

In many different fields of application, such as laser radar, squeezed-state generation, spectroscopy,

0003-6935/08/326022-11\$15.00/0

(C) 2008 Optical Society of America metrology, and gravitational wave detection (see, e.g., the references in [11]), NPROs are used because of their high stability and reliability. In experiments with high-power requirements they are often used as the master laser for amplifiers $[12,13]$ or in injection-lock configurations [14] where the frequency stability of the whole laser system is determined by the master laser.

In the second-generation gravitational wave detector Advanced LIGO [15] an NPRO is used as the master laser in a three-stage laser system concept [16]. Six identical prestabilized laser systems (three observatory lasers, two spare lasers, and one reference system) that will be continuously operated for several years are needed. In this case the variations among several lasers of the same model and the long-term behavior of different beam properties are of interest, since only then appropriately tolerant stabilizations can be designed.

We have developed a compact general purpose laser beam diagnostic instrument called diagnostic breadboard (DBB). It was a significantly extended and improved version of the instrument described in [17]. The DBB was designed for a characterization of linearly polarized, single-frequency, continuous 
wave laser beams. It allows one to measure power noise, frequency noise, and beam pointing fluctuations in a Fourier frequency band from $1 \mathrm{~Hz}$ to $100 \mathrm{kHz}$ as well as power noise at radio frequencies (RF) up to $100 \mathrm{MHz}$ and spatial beam quality. The laser beam characterization was completely automated by a computer, except for the RF power noise measurement.

We used this automated measurement system to characterize eight NPROs (Mephisto 2000 NE LIGO, Innolight [9]) directly after delivery. Afterwards one NPRO was characterized every $24 \mathrm{~h}$ for more than 2600 h ( $\approx 3.5$ months).

The measurements were used to investigate the variations among the eight lasers concerning the free-running noise of several beam properties and the frequency control actuators. Furthermore we gained information on the long-term behavior of one NPRO concerning the free-running noise and demonstrated the reliability of the DBB as a laser beam characterization instrument.

In the past several experiments were performed to measure the beam properties of NPROs or similar lasers, as for example in combination with a stabilization of the output power $[7,18]$, the frequency $[8,19]$, or the beam quality $[18,20]$. However to our knowledge neither the variation of laser properties over several different NPROs nor the long-term variations were analyzed in detail. In particular we are not aware of any experiments that analyzed the complete set of laser beam properties that are accessible with the DBB.

Most of our characterization results show small variations between the different NPROs. With the data of the beam property variations of the eight lasers and the long-term behavior, tolerant stabilizations can now be built. The inspected NPROs are ideally suited for operation in gravitational wave detectors.

Besides the Advanced LIGO, other next generation gravitational wave detectors [21] and many other precision experiments will use NPROs as light sources and therefore will profit from the extensive characterization that is presented here.

\section{Laser Beam Parameters}

The complex field amplitude $U(\mathbf{r}, t)$ of a singlefrequency, polarized laser beam can be expressed in the paraxial approximation as

$$
U(\mathbf{r}, t)=U_{0}(t) \times \exp [2 \pi i \nu(t) t] \times \sum_{l, m \geq 0} c_{l m}(t) \Phi_{l m}(\mathbf{r}),
$$

where $U_{0}$ is the time-dependent amplitude, $\nu$ is the laser frequency, $\Phi_{l m}$ are the complex amplitudes of the Hermite-Gaussian modes $\mathrm{TEM}_{l m}$, and $c_{l m}$ are the complex expansion coefficients or modal weights. The functions $\Phi_{l m}$ form an orthogonal complete set of functions that depends on the beam axis, waist size, and waist position. The coefficients $c_{l m}$ and the func- tions $\Phi_{l m}$ are normalized with

$$
\int \mathrm{d} x \mathrm{~d} y\left|\Phi_{l m}(\mathbf{r})\right|^{2}=1, \quad \sum_{l, m \geq 0}\left|c_{l m}(t)\right|^{2}=1
$$

The properties of a laser beam can be divided into categories according to the three terms in Eq. (1): the power of the laser beam is determined by $U_{0}(\bar{t})$, the frequency by $\nu(t)$, and the beam geometry by $c_{l m}(t)$.

The relative power fluctuations or relative power noise (RPN, often also called relative intensity noise) can be expressed by

$$
P(t) / P_{0}=\left|U_{0}(t)\right|^{2} / \overline{\left|U_{0}(t)\right|^{2}} .
$$

The beam quality, pointing, ellipticity, and astigmatism can be derived from the beam geometry. The beam quality is measured in a quantity called fundamental power $P_{\text {fund }} / P_{\text {tot }}$. The fundamental power is the ratio of the power $P_{\text {fund }}$ in the TEM 00 mode to the total power $P_{\text {tot }}$, where a fundamental power near $100 \%$ corresponds to a high beam quality,

$$
P_{\text {fund }} / P_{\text {tot }}=\left|c_{00}\right|^{2} / \sum_{l, m}\left|c_{l m}\right|^{2}=\left|c_{00}\right|^{2} \text {. }
$$

In experiments with optical resonators the fundamental power is much more suited to define the beam quality than the commonly used $M^{2}$ value. The optical resonators are often stabilized to a resonance of the $\mathrm{TEM}_{00}$ mode, and the fundamental power is equal to the power fraction that can be coupled to such a resonator. With the $M^{2}$ value only a rough lower limit for the coupling efficiency can be derived [17].

Beam pointing and its fluctuation relative to a given reference axis can be described by the complex quantity $\epsilon(t)$,

$$
\epsilon(t)=\frac{\delta x}{w_{0}}+i \frac{\delta \alpha}{\Theta_{D}},
$$

where $\delta x$ is the transversal shift and $\delta \alpha$ is the tilt of the beam. These parameters are normalized with the beam waist radius $w_{0}$ and the half divergence angle $\Theta_{D}$ of the Gaussian beam. The complex amplitude of such a shifted and tilted beam can be expanded in Hermite-Gauss modes of the reference beam in the $\epsilon \ll 1$ limit [22]:

$$
U\left(\epsilon_{x}, \epsilon_{y}\right) \approx \Phi_{00}+\epsilon_{x} \Phi_{10}+\epsilon_{y} \Phi_{01}
$$

Therefore $\epsilon_{x}(t)$ and $\epsilon_{y}(t)$ can be determined by measuring the relative field amplitudes of the $\mathrm{TEM}_{10}$ and TEM $_{01}$ modes:

$$
\epsilon_{x}(t) \approx c_{10} / c_{00}, \quad \epsilon_{y}(t) \approx c_{01} / c_{00} .
$$

In the same way, the relative ellipticity and/or astigmatism $\mu$ can be described by the Laguerre- 
Gaussian mode $\mathrm{Lag}_{02}$ (superposition of a $\mathrm{TEM}_{20}$ and $\mathrm{TEM}_{02}$ mode),

$$
U(\mu) \approx \Phi_{00}+\mu\left(\Phi_{20}-\Phi_{02}\right) / \sqrt{2} \Rightarrow \mu \approx \frac{c_{20}-c_{02}}{\sqrt{2} c_{00}} .
$$

\section{Methods}

We used an advanced version of the instrument described in [17] to measure the laser beam parameters. The DBB is a compact $\left(61 \times 50 \mathrm{~cm}^{2}\right)$ transportable instrument used to characterize single-frequency, linearly polarized laser beams at a wavelength of $1064 \mathrm{~nm}$. The key component in the optical setup (Fig. 1) of the DBB is an optical ring resonator that was used for frequency, pointing, and beam quality measurements. Four photodetectors were used to measure several properties of the input beam. A crate (19 in., six rack units) contained all necessary electronics except for power supplies. The measurement methods used are briefly described in the following subsections.

\section{A. Power Noise Measurement}

The power of the input beam and the power fluctuations were measured with the photodetector RPD (Fig. 1). The DBB was optimized and calibrated for an operating point with an input power of $135 \pm 15 \mathrm{~mW}$. The photodetector consisted of a $2 \mathrm{~mm}$ InGaAs photodiode, a low-noise high-current transimpedance amplifier, and signal conditioning electronics. In the operation point a photocurrent of $50 \mathrm{~mA}$ was detected with a bandwidth $(-3 \mathrm{~dB}$ point) of $\approx 45 \mathrm{MHz}$. The electronic noise (measured with no light on the photodetector) was at $10 \mathrm{MHz}$, a factor of 5 below the shot-noise level of $50 \mathrm{~mA}$ photocurrent. The electronic noise slowly increased

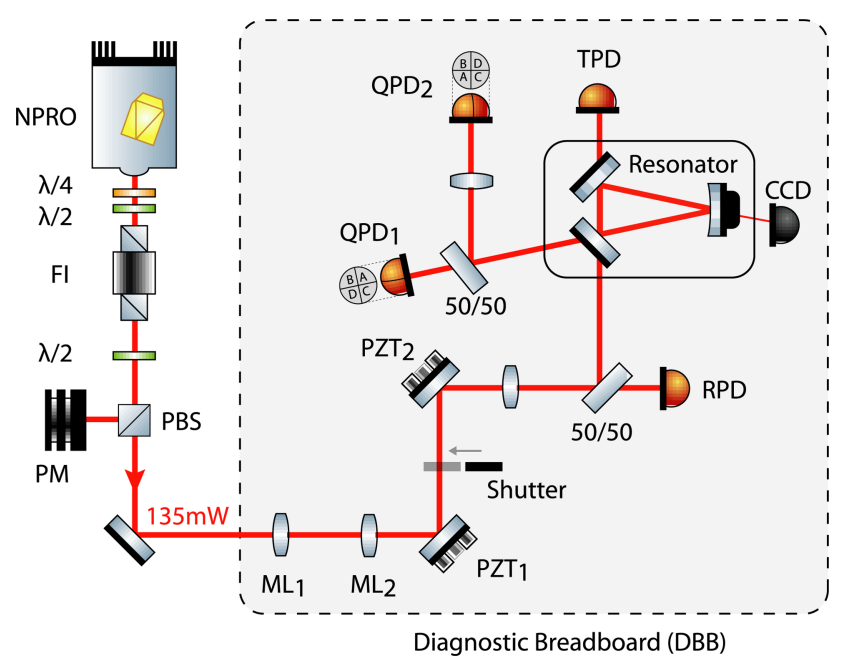

Fig. 1. (Color online) Experimental setup. The NPRO lasers were coupled one after the other to the $\mathrm{DBB}$, where several beam properties were measured. Details of the purpose of the components are given in Sections $3-5$. FI, Faraday isolator; $\lambda / 2$, $\lambda / 4$, waveplates; PBS, polarizing beam splitter; PM, powermeter; $\mathrm{ML}$, mode matching lenses; PZT, mirror with piezoelectric elements; RPD, QPD, TPD, photodetectors. towards higher frequencies and was around $80 \mathrm{MHz}$ at the level of shot noise. The signal conditioning electronics optimized the signal for measurements in the two frequency bands $1 \mathrm{~Hz} \ldots 100 \mathrm{kHz}$ and $1 \mathrm{MHz} . .100 \mathrm{MHz}$.

The shot-noise-limited sensitivity (RPN of $2.5 \times$ $10^{-9} \mathrm{~Hz}^{-1 / 2}$ at $50 \mathrm{~mA}$ ) of the photodetector for frequencies $\geq 5 \mathrm{MHz}$ was verified with a shot-noiselimited test beam. This test beam was prepared by sending an NPRO beam through an optical resonator with a bandwidth of $1 \mathrm{MHz}$, which attenuates the technical power noise to a level below the shot noise of about $70 \mathrm{~mW}$ for these frequencies. In a different experiment the power of the test beam was stabilized for frequencies below $100 \mathrm{kHz}$ using an acousto-optic modulator and a photodetector in front of the DBB. The residual power noise was then measured out-ofloop with the DBB. For frequencies $\geq 200 \mathrm{~Hz}$ the measured relative power noise was approximately $10^{-8} \mathrm{~Hz}^{-1 / 2}$. Towards lower frequencies the noise increased, and at $10 \mathrm{~Hz}$ an RPN of $3 \times 10^{-7} \mathrm{~Hz}^{-1 / 2}$ was measured. These values can serve as an upper limit for the relative power noise sensitivity of the DBB. This sensitivity of the RPD photodetector for frequencies below $100 \mathrm{kHz}$ was at a level that can be lowered only with further efforts such as the use of vacuum tanks or mode cleaners [7]. The measured RPN in the NPRO characterization runs (see Section 6.A) was never limited by the RPD noise.

The signal of the RPD photodetector was used as well to trigger an interlock in case of excessive input power. In such an event a mechanical shutter on the DBB was closed in order to protect the photodetectors.

\section{B. Frequency Noise Measurement}

Frequency fluctuations of the input beam were measured with the optical ring resonator: the resonator was similar to the design described in [18]. A finesse of $\approx 356$ was measured, and a FSR of $\overline{715} \mathrm{MHz}$ was calculated. We were able to electronically adjust the round-trip length of $420 \mathrm{~mm}$ by about $5 \mu \mathrm{m}$ with a piezoelectric element. The resonator was placed inside an aluminum tank for acoustic shielding and to avoid contamination of the resonator mirrors.

The resonator was stabilized to the frequency of the input beam using a dither lock: The round-trip length of the resonator was modulated at $1 \mathrm{MHz}$ with the piezoelectric element in order to create sidebands inside the resonator. The input beam and the phasemodulated beam from the resonator were superposed at the input coupling mirror and were detected with photodetector $\mathrm{QPD}_{1}$. The demodulated signal of this photodetector was used as error signal for the control of the resonator round-trip length. The resonator was stabilized to a resonance of the fundamental mode with a bandwidth of a few $\mathrm{kHz}$ using a feedback control loop and the piezoelectric element.

Frequency fluctuations of the input beam were composed from the control and error signal in a Fourier frequency band from $1 \mathrm{~Hz}$ to $100 \mathrm{kHz}$. The 
dither-lock frequency of $1 \mathrm{MHz}$ limited the bandwidth of the error signals to about $100 \mathrm{kHz}$. The spacer of the resonator was manufactured of aluminum and its stability as frequency reference was limited for Fourier frequencies below $1 \mathrm{~Hz}$ by environment temperature fluctuations. The FSR of the resonator was calculated from the macroscopic round-trip length in order to calibrate the control signal at the piezoelectric element.

With a frequency stabilized test beam the sensitivity for frequency fluctuations was measured. The test beam was stabilized to a high-finesse invacuum reference cavity made of ultralow expansion (ULE) glass. The apparent frequency fluctuations measured with the DBB, and hence the sensitivity of the frequency noise measurement, were below the free-running frequency noise of an NPRO $\left(10 \mathrm{kHz} \mathrm{Hz}^{1 / 2} / f\right.$ [19]). Additional investigations showed that the tank on the DBB significantly improved the stability of the resonator as frequency reference. Compared with a closed tank the measured frequency fluctuations increased with an open one in some frequency bands by two to three orders of magnitude. In a further experiment the tank was evacuated, but the sensitivity of the measurement system was unaffected. Thus a closed tank at atmospheric pressure was used for all further experiments.

\section{Spatial Fluctuations and Beam Quality Measurements}

We measured the pointing fluctuations of the input beam by using the differential wavefront sensing (DWS) and the ring resonator as the pointing reference $[17,22,23]$. Pointing deviations between the fundamental mode, to which the resonator was stabilized, and the input beam were measured. Two quadrant photodetectors, $\mathrm{QPD}_{1}$ and $\mathrm{QPD}_{2}$, were used to detect the DWS signals. The four degrees of freedom, translation and tilt in horizontal and vertical direction (labeled $1 X, 1 Y, 2 X, 2 Y$ in the following), were measured in order to calculate the quantities $\epsilon_{x}$ and $\epsilon_{y}$. Dependent on the considered position along the beam axis, the $1 X / 1 Y$ degree can correspond to a translation, tilt, or mixture of both. At the position of mirror $\mathrm{PZT}_{1}$ and photodiode $\mathrm{QPD}_{1}$ the $1 X / 1 Y$ degree correspond to a pure tilt of the beam. Accordingly the $2 X / 2 Y$ degree corresponds to a pure tilt at mirror $\mathrm{PZT}_{2}$ and photodiode $\mathrm{QPD}_{2}$. Anyhow all four degrees of freedom were orthogonal.

The detectors $\mathrm{QPD}_{1}$ and $\mathrm{QPD}_{2}$ each consisted of a silicon quadrant photodiode and four transimpedance amplifiers. The DWS detection had a bandwidth of about $100 \mathrm{kHz}$.

In order to stay in the linear range of the DWS signals, the pointing of the input beam was adjusted in servo loops using two mirrors $\left(\mathrm{PZT}_{1}, \mathrm{PZT}_{2}\right)$ that could be tilted with piezoelectric elements. The four servo loops had a bandwidth of a few $100 \mathrm{~Hz}$, and the tilt range of $\mathrm{PZT}_{1}$ and $\mathrm{PZT}_{2}$ was about $4 \mathrm{mrad}$, which corresponds to $\epsilon \approx 6$ (see Section 2). A welcome side effect was that the servo loops automatically compensated misalignments introduced by moving lenses during the process of manually mode matching the input beam to the resonator fundamental mode.

The control signals at the piezoelectric elements were calibrated using the resonator: The power of the first-order TEM modes that are excited in the resonator by a misaligned $\mathrm{TEM}_{00}$ input beam depend on the input beam pointing $\epsilon$ with

$$
\frac{\left|c_{10}\right|^{2}}{\left|c_{00}\right|^{2}}=\left|\epsilon_{x}\right|^{2}, \quad \frac{\left|c_{01}\right|^{2}}{\left|c_{00}\right|^{2}}=\left|\epsilon_{y}\right|^{2} .
$$

For calibration, this power ratio was measured using the mode scan technique [17] while varying the alignment control signals.

The beam quality of the input beam was measured with the mode scan technique: The input beam was expanded for this analysis into the eigenmodes of the ring resonator in order to determine the power in higher-order TEM modes. The eigenmodes of the resonator were in very good approximation the Hermite-Gaussian TEM modes. The resonator round-trip length was changed by several micrometers within a second with a ramp signal at the piezoelectric element. The transmitted power was simultaneously measured with photodetector TPD. Since the TEM modes had different resonance frequencies (except for certain degeneracies) we were able to measure the power in the individual modes. The measurements were automatically analyzed by a computer program that identified the modes using their resonance frequencies. The ellipticity and/or astigmatism of the input beam was then determined from the power in the $\mathrm{Lag}_{02}$ mode.

The photodetector TPD consisted of a $2 \mathrm{~mm}$ InGaAs photodiode with a low-offset transimpedance amplifier and saturable DC coupled amplifiers for signal conditioning. The detector was optimized for linearity and was shielded from stray light in the optical setup.

The sensitivity of the measurement method was determined using a single-mode fiber-coupled test beam that was further filtered by a resonator with a finesse of about 350 in front of the DBB. The determined power in higher modes was $0.6 \pm 0.3 \%$ in this experiment. Thus the sensitivity was sufficient to measure the beam quality of the NPRO lasers that had a few percent power in higher modes (see Section $\underline{6 . C \text { ). }}$.

\section{Automation}

The DBB was designed to be remotely controllable. Thus we were able to electronically adjust the alignment of the input beam with two mirrors with piezoelectric elements $\left(\mathrm{PZT}_{1}, \mathrm{PZT}_{2}\right)$ and to optimize the mode matching with motorized lenses $\left(\mathrm{ML}_{1}, \mathrm{ML}_{2}\right)$. All settings that could be made with the front panel controls of the electronic modules could be overridden by analog and digital signals on the crate bus. 
Furthermore all measurement signals were available on this bus.

A module in the electronic crate served as interface between the crate bus and a laboratory computer. Several digital input/output channels and digitalto-analog converters were connected via an $\mathrm{I}^{2} \mathrm{C}$ bus and a RS-232 link with the computer in order to read and write digital signals and to set slow analog signals on the bus. The actual analog measurement signals were distributed with a multiplexer to four channels. Each channel consisted of a signal conditioning unit and an anti-aliasing filter. These conditioned signals were simultaneously digitalized with an analog-to-digital converter card in the computer (PCI-6122, National Instruments) at a sampling rate of $250 \mathrm{kHz}$ and a resolution of 16 bits. A fast analog signal injection for measuring transfer functions was realized with a digital-to-analog card (PCI-6251, National Instruments).

Almost all measurements with the DBB were automized with programs and scripts (except for RPN measurements at RF since the bandwidth of the anti-aliasing filters and the analog-to-digital converter card was limited to about $100 \mathrm{kHz}$ ). The following steps were automatically performed in a usual measurement run: at first the alignment of the input beam was optimized using the DWS signals and the alignment feedback control system. After this step 200 mode scans with about $24 \mathrm{k}$ samples per free spectral range (FSR) were measured and analyzed. The average total power in higher modes and the average power in each identified mode were determined. Then the RPN was measured by sampling the time signal of the photodetector RPD for $1800 \mathrm{~s}$. The linear spectral density (LSD) for Fourier frequencies from $1 \mathrm{~Hz}$ to $100 \mathrm{kHz}$ was calculated to characterize the noise. The photodetector signal was downsampled to $1 \mathrm{~Hz}$ for characterizing the power trend and to calculate the root-mean-square (rms) value for power fluctuations between $0.6 \mathrm{mHz}$ and $1 \mathrm{~Hz}$. Afterwards the resonator of the DBB was stabilized to the input beam in order to measure frequency noise. A transfer function from the calibrated control signal at the ring resonator piezoelectric element to the error signal of the servo loop was measured to calibrate the error signal. Then the error signal and the control signal were simultaneously sampled for $1800 \mathrm{~s}$. The LSD of both signals was calculated and merged to one LSD for Fourier frequencies between $1 \mathrm{~Hz}$ and $100 \mathrm{kHz}$ using the transfer function of the servo loop. The pointing fluctuations were measured consecutively for each degree of freedom. For calibration of the error signals, transfer functions from the calibrated control signals to the error signals were measured. As for the frequency noise measurement the error and control signals were sampled for $1800 \mathrm{~s}$ and an LSD of the pointing fluctuations was calculated. The control signal was further used to measure the pointing at a rate of $1 \mathrm{~Hz}$ and to calculate the rms value of pointing fluctuations from $0.6 \mathrm{mHz}$ to $1 \mathrm{~Hz}$.
By using this control and data acquisition system (CDS) we were able to fully automate the beam characterization, which took about $3.75 \mathrm{~h}$ for one run.

One of the eight lasers was operated continuously and was automatically characterized with this system every $24 \mathrm{~h}$ for a period of over $2600 \mathrm{~h}$ ( $\approx 3.5$ months). The CDS started the measurement run every day at $1 \mathrm{am}$. Except for a few runs, all measurements were successfully completed, and in the end 110 laser beam characterizations were performed. The power fraction coupled into the DBB and the mode matching of the input beam to the DBB resonator were adjusted only during the initial setup and then were left unchanged for the whole 3.5 month measurement period. Thus we were able to measure the long-term variations of the beam power and the mode matching.

\section{Laser}

Eight lasers of the same model (Mephisto $2000 \mathrm{NE}$ LIGO, Innolight) were characterized with the DBB. The lasers, in the following also designated A to $\mathrm{H}$, were solid-state Nd:YAG NPROs with an output power of $2 \mathrm{~W}$ at a wavelength of $1064 \mathrm{~nm}$. The active medium was pumped by laser diodes at a wavelength of $808 \mathrm{~nm}$. The lasers had single-frequency emission and a very good frequency stability due to the monolithic laser resonator/crystal. The laser frequency was controllable with a piezoelectric element at the laser resonator and via the laser crystal temperature. The output beam was slightly elliptically polarized, and the beam quality was specified with $M^{2}<1.1$ from the manufacturer. The lasers came with a built-in power stabilization called Noise Eater for suppressing the relaxation oscillation.

The complete optical setup (Fig. 1) was mounted on a different breadboard $\left(75 \times 90 \mathrm{~cm}^{2}\right)$ in order to be transportable. A $\lambda / 2$ and a $\lambda / 4$ waveplate were used directly behind the laser aperture to convert the elliptically polarized beam to a linearly polarized one. The Faraday isolator (FI) protected the laser from spurious backreflections. Afterwards about $135 \mathrm{~mW}$ were coupled out with a combination of $\lambda / 2$ waveplate and polarizing beam splitter for characterization with the DBB. The rest of the power was detected with a powermeter (PM). The measurements were performed in three different laboratories on optical tables with slow-running clean-air flowboxes without seismic isolation.

All lasers were characterized directly after delivery, and further on laser $\mathrm{H}$ was periodically characterized in the long-term measurement. Some laser-specific properties were measured additionally to the properties described in Section 3: the laser output power was measured with a PM as a function of the pump laser diode current, the so-called output power slope. Then the power remaining downstream of the FI was measured. With this measurement losses due to the waveplates, the FI and the depolarization of the NPRO can be deduced. The frequency of the relaxation oscillation was determined during 
the measurement of the RPN at RF. Finally the transfer functions from the piezoelectric element frequency actuator and the laser crystal temperature to the laser frequency was measured with the DBB.

\section{Results}

An overview of all performed measurements, which are divided into measurements during the initial characterization of all eight lasers and the long-term measurement of laser $\mathrm{H}$, is given in Table 1 . A selection of the results is presented in Figs. 2 to 11 , and some important results of the initial characterization are summarized in Table $\underline{2}$.

\section{A. Power Noise Investigations}

All lasers but laser B had a total output power of about $2.2 \mathrm{~W}$ (Table 2) when the lasers were operated slightly below their individual pump diode current limit. With the cleaning of the polarization state by one polarization beam splitter of the FI and the losses of the FI itself, the output power was reduced to about $1.8 \mathrm{~W}$ to $1.9 \mathrm{~W}$-except for laser $\mathrm{B}$, which delivered only about $1.6 \mathrm{~W}$.

The output power of laser $\mathrm{H}$ degraded slowly during the long-term operation. This degradation was expected because of the aging of the pump laser diodes. Since the laser was operated so close to its current limit, the laser diode current could not be used to compensate for this effect. The degradation had a slope of about $-5 \times 10^{-4}$ /day or -0.18 /year during the monitored operation time of about $2600 \mathrm{~h}$. A slowdown of this degradation in the last $1000 \mathrm{~h}$ of the long-term characterization was observed. Further monitoring of the output power with a PM from operating hour 4000 to 6500 verified a slowdown to $-2 \times 10^{-5}$ /day or $-0.01 /$ year.

With the degradation of the output power the beam width changed as well. This caused a further power reduction of the fundamental mode since

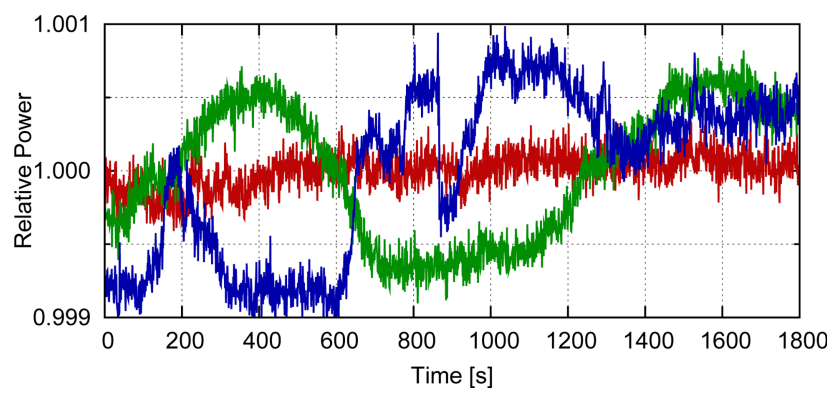

Fig. 2. (Color online) Three different output power time series from the long-term characterization of laser H. The curves show the different types of fluctuations.

the mode matching was not reoptimized from time to time. The mode matching changed during the long-term characterization with $6.6 \times 10^{-5} /$ day (see Section 6.C). Since the fundamental power depends quadratically on the mode matching this additional effect can be neglected when one is explaining the output power degradation.

A histogram of the power fluctuations was calculated and showed a non-Gaussian distribution. The samples for the histogram were acquired at a rate of $1 \mathrm{~Hz}$ during 83 intervals, each $1800 \mathrm{~s}$ long. Since the average power was calculated for every $1800 \mathrm{~s}$ section, the frequencies covered reached from $1 / 1800 \mathrm{~Hz}=0.6 \mathrm{mHz}$ to $1 \mathrm{~Hz}$. The wings of the distribution did not decline as fast as those of a Gaussian distribution. Three example time series (Fig. 2) show that the power could stay almost constant, was changing slowly, or even changed with steplike functions. One should be prepared for the possibility that the relative output power can change by about $1 \times 10^{-3}$ in a few seconds. The variation of relative $\mathrm{rms}$ power fluctuations among the different lasers was rather high. The rms value reached from about $150 \times 10^{-6}$ up to $900 \times 10^{-6}$ (Table $\underline{2}$ ).

Table 1. Measurements Performed for the Initial Characterization of All Eight Lasers and for the Long-Term Characterization of Laser $\mathrm{H}$

\begin{tabular}{|c|c|c|}
\hline Measurement & Initial & Long-Term \\
\hline Output power & Table $\underline{2}$ & \\
\hline Output power fluctuations, rms $0.6 \mathrm{mHz} \ldots 1 \mathrm{~Hz}$ & Table 2 & Fig. 2 \\
\hline RPN, $1 \mathrm{~Hz} \ldots 100 \mathrm{kHz}$, with Noise Eater & Fig. $\underline{3}$ & Fig. $\overline{\underline{4}}$ \\
\hline RPN, $1 \mathrm{~Hz} \ldots 100 \mathrm{kHz}$, w/o Noise Eater & - & \\
\hline Frequency noise, $1 \mathrm{~Hz} \ldots 100 \mathrm{kHz}$ & - & Fig. $\underline{6}$ \\
\hline Frequency noise trend at selected frequencies & & Fig. $\overline{\overline{7}}$ \\
\hline Piezo element frequency actuator & Table $\underline{2}$ & \\
\hline Temperature frequency actuator & Table $\underline{2}$ & \\
\hline Pointing fluctuations, $\mathrm{rms} 0.6 \mathrm{mHz} \ldots 1 \mathrm{~Hz}$ & Table $\underline{\overline{2}}$ & Fig. $\underline{8}$ \\
\hline Pointing fluctuations, $1 \mathrm{~Hz} \ldots 100 \mathrm{kHz}$ & Fig. $\underline{9}$ & - \\
\hline
\end{tabular}


Table 2. Several Beam and Frequency Actuator Properties Measured During the Initial Characterization

\begin{tabular}{lccccccccc}
\hline Laser & $\mathrm{A}$ & $\mathrm{B}$ & $\mathrm{C}$ & $\mathrm{D}$ & $\mathrm{E}$ & $\mathrm{F}$ & $\mathrm{G}$ & $\mathrm{H}$ & Average and Standard Deviation \\
\hline Total output power $(\mathrm{mW})$ & 2250 & 2010 & 2230 & 2160 & 2210 & 2200 & 2270 & 2230 & $2195 \pm 76$ \\
Output power after FI $(\mathrm{mW})$ & 1940 & 1560 & 1860 & 1870 & 1900 & 1940 & 1920 & 1930 & $1865 \pm 119$ \\
Output power fluctuation, relative $\mathrm{rms}\left(10^{-6}\right)$ & 875 & 186 & 777 & 252 & 394 & 154 & 428 & 460 & $441 \pm 247$ \\
Relaxation oscillation frequency $(\mathrm{kHz})$ & 1230 & 1210 & 1210 & 900 & 1160 & 975 & 1230 & 1200 & $1139 \pm 120$ \\
Piezo frequency actuator calibration $(\mathrm{MHz} / \mathrm{V})$ & 1.60 & 1.26 & 1.70 & 1.33 & 1.26 & 1.45 & 1.26 & 2.00 & $1.48 \pm 0.25$ \\
Temperature frequency actuator calibration $(\mathrm{GHz} / \mathrm{K})$ & 3.4 & 2.8 & 2.8 & 2.7 & 2.8 & 3.0 & 2.5 & 2.5 & $2.8 \pm 0.3$ \\
Temperature frequency actuator bandwidth $(\mathrm{mHz})$ & 90 & 200 & 142 & 220 & 130 & 150 & 200 & 175 & $163 \pm 41$ \\
Pointing fluctuation $1 X$, relative $\mathrm{rms}\left(10^{-3}\right)$ & 6.6 & 6.6 & 4.0 & 8.1 & 4.6 & 3.6 & 3.6 & 6.9 & $5.5 \pm 1.6$ \\
Pointing fluctuation $1 Y$, relative $\mathrm{rms}\left(10^{-3}\right)$ & 8.7 & 9.8 & 2.8 & 11.5 & 2.5 & 2.8 & 2.1 & 9.0 & $6.1 \pm 3.7$ \\
Pointing fluctuation $2 X$, relative $\mathrm{rms}\left(10^{-3}\right)$ & 4.1 & 5.4 & 1.8 & 5.4 & 1.8 & 1.4 & 1.9 & 4.5 & $3.3 \pm 1.6$ \\
Pointing fluctuation $2 Y$, relative rms $\left(10^{-3}\right)$ & 6.2 & 7.4 & 1.6 & 7.9 & 1.1 & 1.3 & 1.8 & 6.6 & $4.2 \pm 2.8$ \\
Beam quality (higher mode content) $(\%)$ & 2.43 & 2.37 & 1.39 & 4.37 & 1.37 & 3.39 & 2.23 & 2.28 & $2.49 \pm 1.00$ \\
Relative ellipticity/astigmatism & 0.065 & 0.073 & 0.059 & 0.151 & 0.013 & 0.148 & 0.072 & 0.089 & $0.084 \pm 0.043$ \\
\hline
\end{tabular}

The linear spectral density of the RPN was almost flat at a level of $5 \times 10^{-7} \mathrm{~Hz}^{-1 / 2}$ from $10 \mathrm{~Hz}$ to $100 \mathrm{kHz}$ with Noise Eater disabled (Fig. 3). The differences between the eight lasers were small. Below $10 \mathrm{~Hz}$ the RPN was increasing very fast towards lower frequencies with more than one order of magnitude per decade. A comparison to measurements with activated Noise Eater showed that this power stabilization reduced the noise level starting from about $100 \mathrm{~Hz}$ (Fig. 3). Above around $300 \mathrm{~Hz}$ the RPN with Noise Eater was almost flat at a level of $\approx 2 \times 10^{-7} \mathrm{~Hz}^{-1 / 2}$. There was no significant difference of the RPN below $10 \mathrm{~Hz}$ with or without Noise Eater. The exemplar variations were rather small for measurements with Noise Eater.

In all measurements there were visible sharp peaks at $50 \mathrm{~Hz}, 150 \mathrm{~Hz}$, and higher harmonics due to the mains frequency of $50 \mathrm{~Hz}$. These undesired signals coupled into the measurement primarily at the CDS system. The photodiode signal itself did not contain these frequencies-this was checked with a battery-powered oscilloscope with fast Fourier transform capability.

As can be seen from the long-term measurements, there were a couple of outliers that were almost an

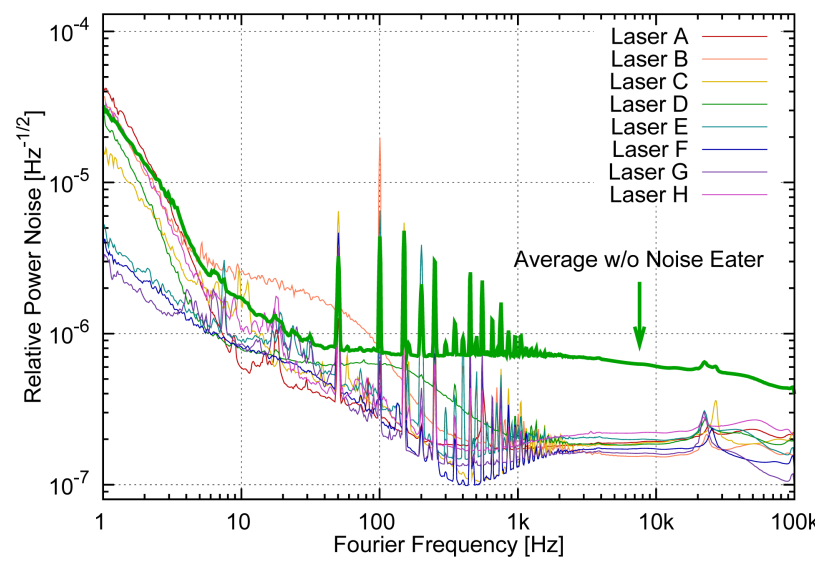

Fig. 3. (Color online) Relative power noise during the initial characterizations of all eight lasers with Noise Eater activated. The average power noise level without Noise Eater is shown for comparison. order of magnitude above the median RPN level at some frequencies (Fig. 4). Especially for frequencies around and above $100 \mathrm{~Hz}$ some measurements strongly differed from the median. Over some period the air particle count was monitored during the longterm measurements. The average particle count of $0.7 \mathrm{ft}^{-3}$ with a size of larger than $0.3 \mu \mathrm{m}$ was very low, and no significant correlation between the particle count and the RPN could be observed. According to this, air particles did not seem to cause the outliers in our setup.

For frequencies below $10 \mathrm{~Hz}$ there were almost no outliers. The RPN in this frequency band was reduced significantly after an aluminum enclosure was mounted around the DBB. This might be an indication that pointing was a dominant noise source at these frequencies, since the pointing fluctuations were reduced by this as well (see Section 6.C). All in all the RPN with Noise Eater at frequencies above $10 \mathrm{~Hz}$ was between $1 \times 10^{-7} \mathrm{~Hz}^{-1 / 2}$ and $1 \times$ $10^{-6} \mathrm{~Hz}^{-1 / 2}$ except for some outliers.

The relaxation oscillation of six lasers was at a frequency of $1.2 \mathrm{MHz}$ (Table 2). Laser $\mathrm{D}$ had a relaxation oscillation frequency at $900 \mathrm{kHz}$, and laser $\mathrm{F}$ at $975 \mathrm{kHz}$. With Noise Eater activated this oscillation was suppressed by a factor of about 50 , and the

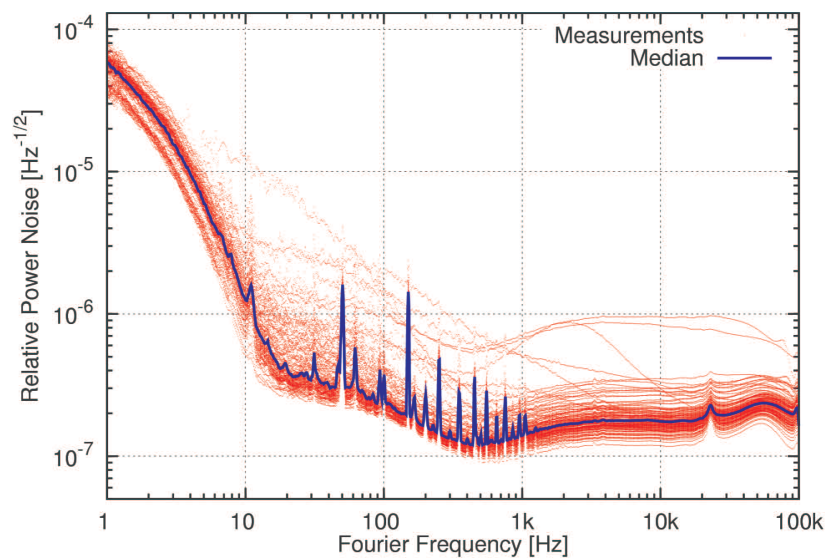

Fig. 4. (Color online) Relative power noise during the long-term characterization of laser $\mathrm{H}$. The median power noise of the 110 measurements is shown for reference. 
RPN level was at about $2 \times 10^{-7} \mathrm{~Hz}^{-1 / 2}$ (Fig. 5). Between $2 \mathrm{MHz}$ and $10 \mathrm{MHz}$ the Noise Eater slightly degraded the RPN, which was, however, still decreasing very rapidly with increasing frequency. For frequencies above $20 \mathrm{MHz}$ the RPN was at or below the shot noise of about $50 \mathrm{~mA}$ photocurrent.

At first the Noise Eater of laser A was increasing the RPN at around $2.5 \mathrm{MHz}$ significantly. This problem was solved by an engineer from the manufacturer who adjusted some parameters of the Noise Eater feedback loop.

\section{B. Frequency Noise Investigations}

As expected the frequency noise of the lasers decreased with $1 / f$ towards higher Fourier frequencies with a value of about $10 \mathrm{kHz} \mathrm{Hz}^{-1 / 2} \cdot[1 \mathrm{~Hz} / f]$. The variations between the different lasers were small, although they were increasing below $10 \mathrm{~Hz}$

The long-term measurement of the frequency noise showed that the noise seemed to be very stationary and that the variations between the measurements were small (Figs. 6 and 7). Compared to the RPN long-term measurement there were clearly fewer outliers from the median. The variations between the long-term measurements and the ones between the different lasers were in the same range. The sharp peaks between $10 \mathrm{kHz}$ and $40 \mathrm{kHz}$ were due to calibration inaccuracies since several resonances of the resonator piezoelectric element were in this frequency band. The spectra also contained peaks at the mains frequency of $50 \mathrm{~Hz}$ and higher odd harmonics. It is hard to determine whether the laser frequency contained these signals or they were caused by detection problems since the dither-lock method used involved several interconnected electronic modules. Since the peaks in the RPN were induced by the CDS system, it is more likely that the peaks in the frequency noise were caused by the dither-lock electronics.

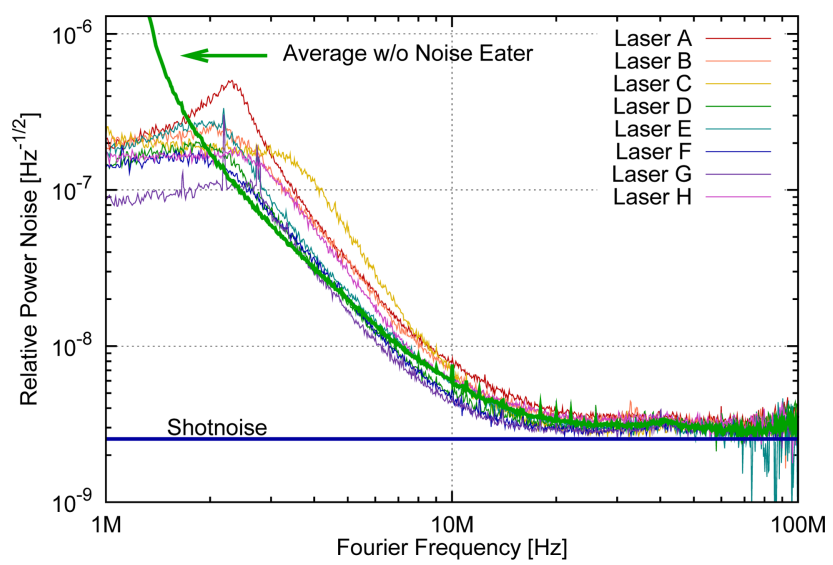

Fig. 5. (Color online) Relative power noise at radio frequencies with Noise Eater activated during the initial characterizations. For comparison the average power noise level without Noise Eater and the shot noise of the detected photocurrent of $50 \mathrm{~mA}$ are shown. For frequencies above about $80 \mathrm{MHz}$ the variance increased since the subtracted electronics noise was at the shot-noise level.

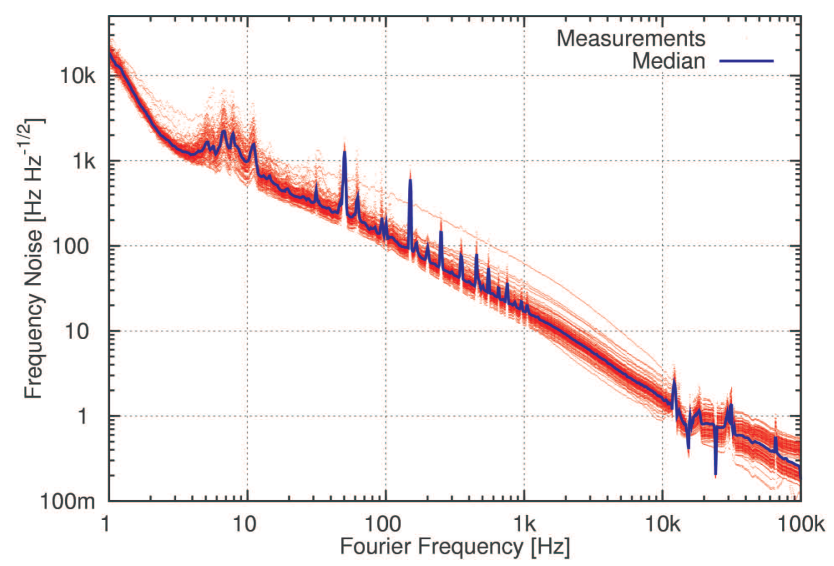

Fig. 6. (Color online) Frequency noise during the long-term characterization of laser $\mathrm{H}$. The median frequency noise of the 110 measurements is shown for reference.

The tuning coefficient of the piezoelectric element at the NPRO crystal turned out to be between $1 \mathrm{MHz} / \mathrm{V}$ and $2 \mathrm{MHz} / \mathrm{V}$ (Table 2). With a maximum voltage of $\pm 100 \mathrm{~V}$ (specified by the manufacturer) the NPRO frequency can be tuned from about $200 \mathrm{MHz}$ to $400 \mathrm{MHz}$ neglecting any nonlinearities. The transfer function was flat in the measurement frequency band $(1 \mathrm{~Hz}$ to $100 \mathrm{kHz})$ of the DBB. To determine the first resonance frequency, the electrical impedance of one piezoelectric element was measured. It had a capacitance of $2 \mathrm{nF}$ and the first resonance at about $200 \mathrm{kHz}$.

The tuning coefficient of the crystal temperature for all lasers was about $2.8 \mathrm{GHz} / \mathrm{K}$ (Table 2). The temperature could be changed by about $3 \mathrm{~K}$ without a mode hop. According to this result a tuning range of about $8.4 \mathrm{GHz}$ could be achieved. The bandwidth of this actuator turned out to be significantly smaller than one would expect from the NPRO manual. The average $-3 \mathrm{~dB}$ bandwidth was about $160 \mathrm{mHz}$. The transfer function seemed to be a first-order low pass with some delay, since the phase was decreasing faster compared to the magnitude.

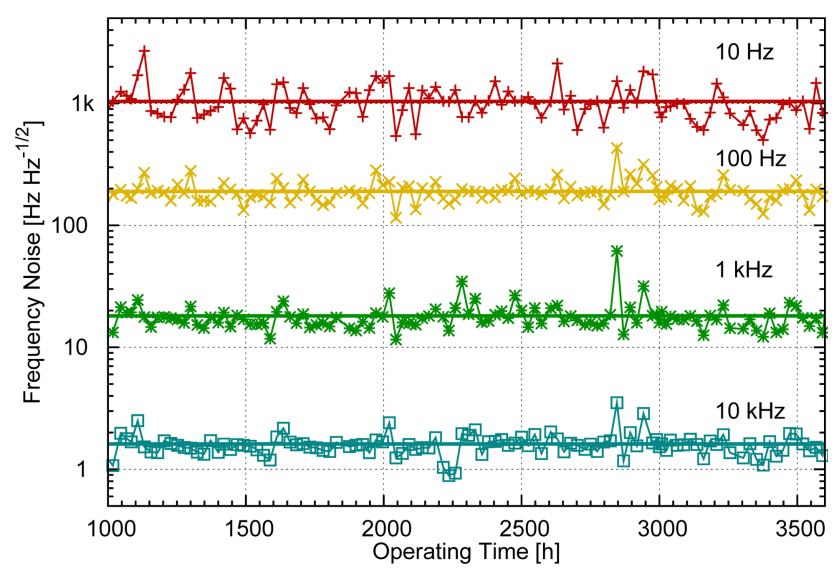

Fig. 7. (Color online) Frequency noise trend at four selected Fourier frequencies during the long-term characterization of laser $\mathrm{H}$. Horizontal lines denote the average noise level. 


\section{Spatial Fluctuations and Beam Quality Investigations}

The histograms of the pointing fluctuations during the long-term measurements could be approximated very well by Gaussian distributions, and thus the distributions were described by the standard deviation or the rms value (Fig. 8). The pointing fluctuations in the $1 X$ and $1 Y$ directions were stronger compared to the $2 X$ and $2 Y$ directions. The fluctuations in the $Y$ directions were slightly stronger than in the $X$ directions. All in all the distributions had a standard deviation of about $5 \times 10^{-3}$. The measured pointing fluctuations of lasers $\mathrm{E}, \mathrm{F}$, and $\mathrm{G}$ were exceptionally low (Table 2).

The pointing noise below $20 \mathrm{~Hz}$ (Fig. 9) seemed to be dominated by the characterization environment. The eight lasers were characterized at three different locations, and especially in the $2 Y$ direction there were three groups of noise levels. Above $20 \mathrm{~Hz}$ the variation between the lasers was relatively large, up to about one order of magnitude. The relative pointing noise level was about $1 \times 10^{-6} \mathrm{~Hz}^{-1 / 2}$.

The variation during the long-term measurement was in the same order of magnitude as the variation between the different lasers. At frequencies below $20 \mathrm{~Hz}$ the variation was rather small, and for frequencies above $20 \mathrm{~Hz}$ the variation was almost one order of magnitude. Above $3 \mathrm{kHz}$ the measurements were limited by electronic noise.

The environment dependence of the measurements became apparent at operating hour 2974 during the long-term measurements. At this time the enclosure was mounted around the DBB. From that moment on the noise level dropped at frequencies below $20 \mathrm{~Hz}$ At frequencies above $20 \mathrm{~Hz}$ the noise level did not change significantly except for the $1 Y$ direction, where the noise level increased slightly.

The interpretation of the pointing measurement results is rather difficult since the environment parameters that influenced the measurements are not known. The pointing was measured at different flowbox speeds. The pointing noise increased at low frequencies with the flow speed of the flowbox. Broadband acoustic coupling could be ruled out as the dominant noise source in further experiments with a loudspeaker.

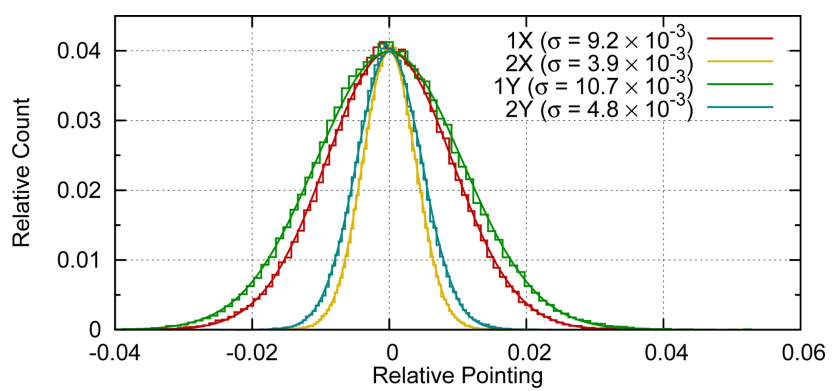

Fig. 8. (Color online) Histogram of pointing fluctuations during the long-term characterization of laser $\mathrm{H}$ (vertical lines between the bins were omitted for clarity). For each degree of freedom $146 \times$ $10^{3}$ samples were evaluated. The standard deviation is shown as a continuous line.

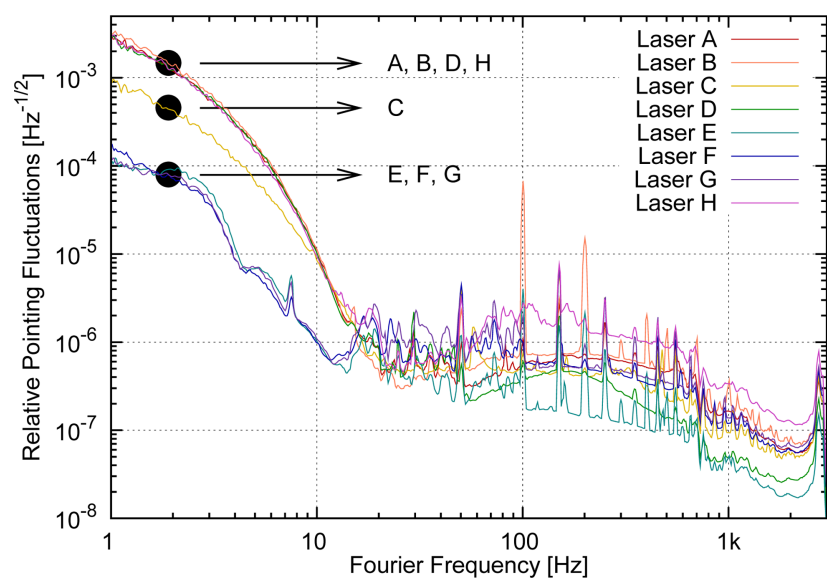

Fig. 9. (Color online) Pointing fluctuations in $2 Y$ direction during the initial characterizations. The lasers were characterized at three different locations in the following groups: lasers A, B, D, $\mathrm{H}$; laser C; and lasers E, F, G.

The lasers had an average of $2.5 \%$ of their power in higher-order TEM modes (Table 2). There were four outliers: Lasers $\mathrm{D}$ and $\mathrm{F}$ had a lower beam quality, and lasers $\mathrm{E}$ and $\mathrm{C}$ had a better one. The most powerful higher modes had a mode order of 2 $\left(\mathrm{TEM}_{02}, \mathrm{TEM}_{20}\right.$; at $\left.0.3 \mathrm{FSR}\right)$ and $4\left(\mathrm{TEM}_{04}\right.$, $\mathrm{TEM}_{22}, \mathrm{TEM}_{40}$; at $0.6 \mathrm{FSR}$ ), all with even $l$-mode numbers $\left(c_{l m}\right.$; Fig. 10). Besides these dominant modes there were many low-power modes in the laser beam. During the long-term measurements the beam quality changed slowly over time (Fig. 11). The average higher mode power during the longterm measurements was $2.4 \pm 0.06 \%$.

The NPROs had a slightly elliptical beam (a value of $1: 1.1$ is given in the manual). This corresponded to a relative ellipticity/astigmatism of 0.1 . An ellipticity and/or astigmatism of about 0.084 was measured for all lasers except D, E, and F (Table 2); lasers D and F had a relative ellipticity/astigmatism of 0.15 , and laser E, 0.01.

\section{Discussion}

The output beams of eight NPRO lasers were extensively characterized with an automated diagnostic tool. The distribution of beam parameters among

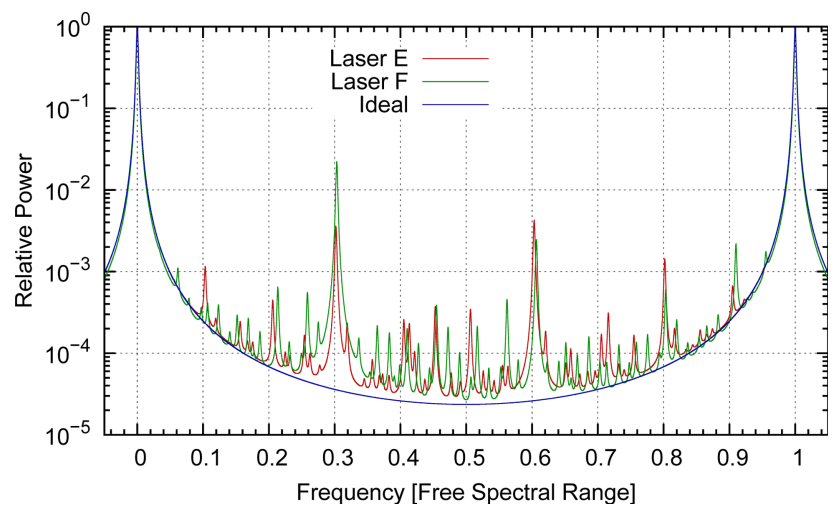

Fig. 10. (Color online) Mode scan of laser $\mathrm{E}$ with the highest and of laser $\mathrm{F}$ with the lowest beam quality. 


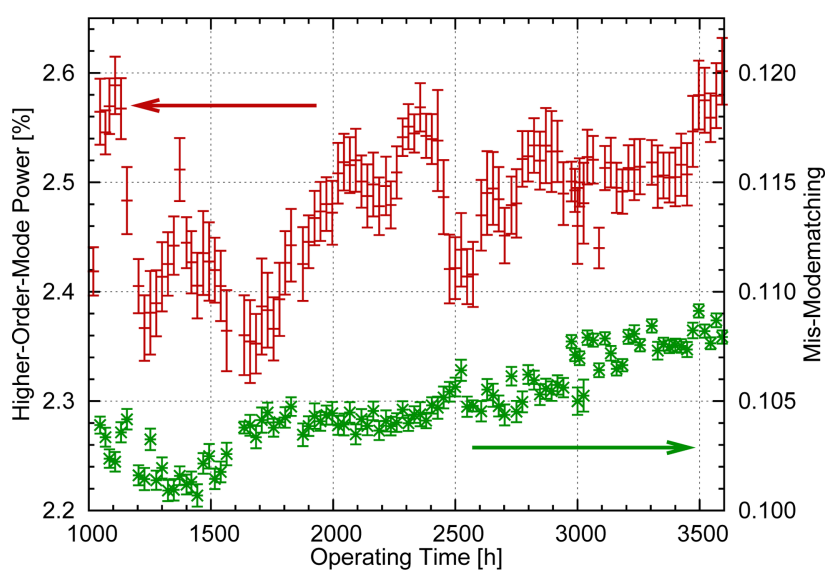

Fig. 11. (Color online) Beam quality trend (measured as higherorder-mode power) and mode mismatching trend during the long-term characterization of laser $\mathrm{H}$.

the eight lasers and the long-term variations were measured. Laser power, frequency, pointing, beam quality, and frequency actuators were characterized. Using these characterization results, suitable and tolerant stabilization schemes can be designed in order to further improve the beam properties.

The characterization results show that NPROs are highly stable laser sources and that the variation between different samples is rather small. This extensive characterization of Nd:YAG NPROs will help in particular precision experiments in selecting a suitable laser beam source. The NPROs are ideal for operation in interferometric gravitational wave detectors. Since they have a low and stationary frequency noise in combination with fast and high dynamic range frequency actuators, they are especially suited as master oscillators for amplifiers or injection-lock configurations if more output power is required. Experiments with optical resonators benefit from the high beam quality and the frequency stability.

The DBB that was used for almost all measurements was reliable and could be operated fully automized for several months. Thus a permanent integration into a laser system is possible in order to automatically monitor the performance and to quickly identify problems. In principle the DBB could be adapted to other laser wavelengths or other Fourier frequency bands. The characterization of the NPROs was not limited by the sensitivity of the DBB except for the pointing fluctuation measurements at high Fourier frequencies. This diagnostic tool can be of great help for developing lasers since the output beam of the laser can be quickly analyzed. Careless mistakes are minimized by the automation of the measurements, and the results are more comparable since each measurement run is performed in the same way.

\section{References}

1. D. Sigg, "Status of the LIGO detectors," Class. Quantum Grav. 23, S51-S56 (2006).
2. D. Tatsumi, R. Takahashi, K. Arai, N. Nakagawa, K. Agatsuma, T. Yamazaki, M. Fukushima, M.-K. Fujimoto, A. Takamori, A. Bertolini, V. Sannibale, R. DeSalvo, S. Marka, M. Ando, K. Tsubono, T. Akutsu, K. Yamamoto, H. Ishitsuka, T. Uchiyama, S. Miyoki, M. Ohashi, K. Kuroda, N. Awaya, N. Kanda, A. Araya, S. Telada, T. Tomaru, T. Haruyama, A. Yamamoto, N. Sato, T. Suzuki, and T. Shintomi, "Current status of Japanese detectors," Class. Quantum Grav. 24, S399-S403 (2007).

3. F. Acernese, P. Amico, M. Alshourbagy, F. Antonucci, S. Aoudia, P. Astone, S. Avino, D. Babusci, G. Ballardin, F. Barone, L. Barsotti, M. Barsuglia, F. Beauville, S. Bigotta, S. Birindelli, M. A. Bizouard, C. Boccara, F. Bondu, L. Bosi, C. Bradaschia, S. Braccini, A. Brillet, V. Brisson, D. Buskulic, E. Calloni, E. Campagna, F. Carbognani, F. Cavalier, R. Cavalieri, G. Cella, E. Cesarini, E. Chassande-Mottin, N. Christensen, C. Corda, A. Corsi, F. Cottone, A.-C. Clapson, F. Cleva, J.-P. Coulon, E. Cuoco, A. Dari, V. Dattilo, M. Davier, M. del Prete, R. D. Rosa, L. D. Fiore, A. D. Virgilio, B. Dujardin, A. Eleuteri, I. Ferrante, F. Fidecaro, I. Fiori, R. Flaminio, J.-D. Fournier, S. Frasca, F. Frasconi, L. Gammaitoni, F. Garufi, E. Genin, A. Gennai, A. Giazotto, G. Giordano, L. Giordano, R. Gouaty, D. Grosjean, G. Guidi, S. Hebri, H. Heitmann, P. Hello, S. Karkar, S. Kreckelbergh, P. L. Penna, M. Laval, N. Leroy, N. Letendre, B. Lopez, M. Lorenzini, V. Loriette, G. Losurdo, J.-M. Mackowski, E. Majorana, C. N. Man, M. Mantovani, F. Marchesoni, F. Marion, J. Marque, F. Martelli, A. Masserot, M. Mazzoni, L. Milano, F. Menzinger, C. Moins, J. Moreau, N. Morgado, B. Mours, F. Nocera, C. Palomba, F. Paoletti, S. Pardi, A. Pasqualetti, R. Passaquieti, D. Passuello, F. Piergiovanni, L. Pinard, R. Poggiani, M. Punturo, P. Puppo, K. Qipiani, P. Rapagnani, V. Reita, A. Remillieux, F. Ricci, I. Ricciardi, P. Ruggi, G. Russo, S. Solimeno, A. Spallicci, M. Tarallo, M. Tonelli, A. Toncelli, E. Tournefier, F. Travasso, C. Tremola, G. Vajente, D. Verkindt, F. Vetrano, A. Vicere, J.-Y. Vinet, H. Vocca, and M. Yvert, "Status of Virgo detector," Class. Quantum Grav. 24, S381-S388 (2007).

4. B. Willke, "GEO600: status and plans," Class. Quantum Grav. 24, S389-S397 (2007).

5. H. Vahlbruch, M. Mehmet, S. Chelkowski, B. Hage, A. Franzen, N. Lastzka, S. Goßler, K. Danzmann, and R. Schnabel, "Observation of squeezed light with 10-db quantum-noise reduction," Phys. Rev. Lett. 100, 033602 (2008).

6. H. Müller-Ebhardt, H. Rehbein, R. Schnabel, K. Danzmann, and Y. Chen, "Entanglement of macroscopic test masses and the standard quantum limit in laser interferometry," Phys. Rev. Lett. 100, 013601 (2008).

7. F. Seifert, P. Kwee, M. Heurs, B. Willke, and K. Danzmann, "Laser power stabilization for second-generation gravitational wave detectors," Opt. Lett. 31, 2000-2002 (2006).

8. F. Bondu, P. Fritschel, C. N. Man, and A. Brillet, "Ultrahighspectral-purity laser for the VIRGO experiment," Opt. Lett. 21, 582-584 (1996).

9. I. Freitag, A. Tünnermann, and H. Welling, "Power scaling of diode-pumped monolithic Nd:YAG lasers to output powers of several watts," Opt. Commun. 115, 511-515 (1995).

10. T. J. Kane and R. L. Byer, "Monolithic, unidirectional singlemode Nd:YAG ring laser," Opt. Lett. 10, 65-67 (1985).

11. T. Day, E. Gustafson, and R. Byer, "Sub-hertz relative frequency stabilization of two-diode laser-pumped Nd:YAG lasers locked to a Fabry-Perot interferometer," IEEE J. Quantum Electron. 28, 1106-1117 (1992).

12. M. Frede, B. Schulz, R. Wilhelm, P. Kwee, F. Seifert, B. Willke, and D. Kracht, "Fundamental mode, single-frequency laser amplifier for gravitational wave detectors," Opt. Express 15, 459-465 (2007). 
13. R. S. Abbott and P. J. King, "Diode-pumped Nd:YAG laser intensity noise suppression using a current shunt," Rev. Sci. Instrum. 72, 1346-1349 (2001).

14. I.Zawischa, M. Brendel, K. Danzmann, C. Fallnich, M. Heurs, S. Nagano, V. Quetschke, H. Welling, and B. Willke, "The GEO600 laser system," Class. Quantum Grav. 19, 1775-1781 (2002).

15. A. Weinstein, "Advanced LIGO optical configuration and prototyping effort," Class. Quantum Grav. 19, 1575-1584 (2002).

16. B. Willke, K. Danzmann, M. Frede, P. King, D. Kracht, P. Kwee, O. Punken, R. Savage, B. Schulz, F. Seifert, C. Veltkamp, S. Wagner, P. Weßels, and L. Winkelmann, "Stabilized high power lasers for advanced gravitational wave detectors," Class. Quantum Grav. 25, 114040 (2008).

17. P. Kwee, F. Seifert, B. Willke, and K. Danzmann, "Laser beam quality and pointing measurement with an optical resonator," Rev. Sci. Instrum. 78, 073103 (2007).

18. B. Willke, N. Uehara, E. Gustafson, R. L. Byer, P. J. King, S. U. Seel, and R. L. Savage, "Spatial and temporal filtering of a $10 \mathrm{~W}$ Nd:YAG laser with a Fabry-Perot ring-cavity premode cleaner," Opt. Lett. 23, 1704-1706 (1998).

19. T. Day, "Frequency-stabilized solid state lasers for coherent optical communications," Ph.D. thesis (Stanford University, 1991).

20. N. Uehara, E. K. Gustafson, M. M. Fejer, and R. L. Byer, "Modeling of efficient mode-matching and thermal-lensing effect on a laser-beam coupling into a mode-cleaner cavity," Proc. SPIE 2989, 57-68 (1997).
21. B. Willke, P. Ajith, B. Allen, P. Aufmuth, C. Aulbert, S. Babak, R. Balasubramanian, B. Barr, S. Berukoff, A. Bunkowski, G. Cagnoli, C. A. Cantley, M. M. Casey, S. Chelkowski, Y. Chen, D. Churches, T. Cokelaer, C. N. Colacino, D. R. M. Crooks, C. Cutler, K. Danzmann, R. J. Dupuis, E. Elliffe, C. Fallnich, A. Franzen, A. Freise, I. Gholami, S. Goßler, A. Grant, H. Grote, S. Grunewald, J. Harms, B. Hage, G. Heinzel, I. S. Heng, A. Hepstonstall, M. Heurs, M. Hewitson, S. Hild, J. Hough, Y. Itoh, G. Jones, R. Jones, S. H. Huttner, K. Kötter, B. Krishnan, P. Kwee, H. Lück, M. Luna, B. Machenschalk, M. Malec, R. A. Mercer, T. Meier, C. Messenger, S. Mohanty, K. Mossavi, S. Mukherjee, P. Murray, G. P. Newton, M. A. Papa, M. Perreur-Lloyd, M. Pitkin, M. V. Plissi, R. Prix, V. Quetschke, V. Re, T. Regimbau, H. Rehbein, S. Reid, L. Ribichini, D. I. Robertson, N. A. Robertson, C. Robinson, J. D. Romano, S. Rowan, A. Rüdiger, B. S. Sathyaprakash, R. Schilling, R. Schnabel, B. F. Schutz, F. Seifert, A. M. Sintes, J. R. Smith, P. H. Sneddon, K. A. Strain, I. Taylor, R. Taylor, A. Thüring, C. Ungarelli, H. Vahlbruch, A. Vecchio, J. Veitch, H. Ward, U. Weiland, H. Welling, L. Wen, P. Williams, W. Winkler, G. Woan, and R. Zhu, "The GEO-HF project," Class. Quantum Grav. 23, S207-S214 (2006).

22. D. Z. Anderson, "Alignment of resonant optical cavities," Appl. Opt. 23, 2944-2949 (1984).

23. E. Morrison, B. J. Meers, D. I. Robertson, and H. Ward, "Automatic alignment of optical interferometers," Appl. Opt. 33, 5041-5049 (1994). 